\title{
Institute for Multiscale Modeling of Biological Interactions
}

\author{
Michael E. Paulaitis and Bertrand Garcia-Moreno, Co-Directors \\ Departments of Chemical/Biomolecular Engineering and Biophysics \\ Johns Hopkins University \\ Abraham Lenhoff, Co-Director \\ Department of Chemical Engineering \\ University of Delaware
}

\section{Institute Goals}

The Institute for Multiscale Modeling of Biological Interactions (IMMBI) has two primary goals:

- Foster interdisciplinary collaborations among faculty and their research laboratories that will lead to novel applications of multiscale simulation and modeling methods in the biological sciences and engineering.

- Building on the unique biophysical/biology-based engineering foundations of the participating faculty, train scientists and engineers to apply computational methods that collectively span multiple time and length scales of biological organization.

\section{Measures of Success}

The success of IMMBI will be defined by the following:

- Size and quality of the applicant pool for pre-doctoral and post-doctoral fellows.

- Academic performance.

- Quality of the pre-doctoral and post-doctoral research.

- Impact of the research broadly and to the DOE (ASCR program) mission.

- Distinction of the next career step for pre-doctoral and post-doctoral fellows.

- Faculty collaborations that result from IMMBI activities.

\section{Institute Accomplishments}

Specific details about accomplishments during the three years of DOE support for IMMBI have been documented in Annual Progress Reports (April 2005, June 2006, and March 2007) and a Report for a National Academy of Sciences Review (October 2005) that were submitted to DOE on the dates indicated. An overview of these accomplishments is provided below.

Our most notable accomplishment was to bring together 26 faculty of all academic ranks from the five research universities - Johns Hopkins, University of Delaware, Ohio State University, University of New Mexico, and Rensselaer Polytechnic Institute - and Los Alamos National Laboratory represented diverse research interests and an extraordinary range of expertise in the biological sciences, biology-based engineering, biophysics, chemistry, physics, statistics, and computational biology. A distinguishing feature of IMMBI that was reflected in the faculty was its strong biophysical/bioengineering foundations upon which the multiscale modeling and computational components of the program were built. The list of participating faculty members, including those added since the inception of the Institute (denoted by *) is given below. 
Johns Hopkins University

Mario Amzel

Dilip Asthagiri*

Joel Bader

Doug Barrick

Greg Chirikjian

David Draper

Karen Fleming*

Bertrand Garcia-Moreno

Jeff Gray

Pablo Iglesias

Andre Levchenko

George Rose

Ingo Ruczinski*

Ben Schafer*

Robert Schleif*

David Shortle*

Sean Sun

Rai Winslow

Tom Woolf

Ohio State University

Michael Paulaitis

University of Delaware

Abraham Lenhoff

Babatunde Ogunnaike

Dion Vlachos*

University of New Mexico

Jeremy Edwards
Biophysics \& Biophysical Chemistry

Chemical \& Biomolecular Engineering

Biomedical Engineering

Biophysics

Mechanical Engineering

Chemistry

Biophysics

Biophysics

Chemical \& Biomolecular Engineering

Electrical and Computer Engineering

Biomedical Engineering

Biophysics

Biostatistics

Civil Engineering

Biology

Biological Chemistry

Mechanical Engineering

Biomedical Engineering

Physiology

Chemical \& Biomolecular Engineering

Chemical Engineering

Chemical Engineering

Chemical Engineering

Chemical Engineering
Medicine

Engineering

Engineering

Arts and Sciences

Engineering

Arts and Sciences

Arts and Sciences

Arts and Sciences

Engineering

Engineering

Engineering

Arts and Sciences

Public Health

Engineering

Arts and Sciences

Medicine

Engineering

Engineering

Medicine

Engineering

Engineering

Engineering

Engineering

Engineering

Science

Rensselaer Polytechnic Institute

Angel Garcia

Physics Applied Physics \& Astronomy

Los Alamos National Laboratory

Lawrence Pratt Theoretical Chemistry \& Molecular Physics Theoretical Division

The IMMBI roster grew by five faculty members since its inception. Two of the original IMMBI members (Neil Clarke at Hopkins and Yong Duan at Delaware) moved to other universities, and decided to no longer be associated or only indirectly associated with the Institute. In addition, Professor Paulaitis moved from Johns Hopkins to Ohio State, Professor Jeremy Edwards moved from the University of Delaware to the University of New Mexico, and Professor Garcia moved from Los Alamos National Laboratory to Rensselaer Polytechnic Institute, but continued to participate in the Institute. During the same period, seven additional faculty members petitioned to become members, and five other faculty - three from Ohio State University, one from the University of Delaware, and one from Rensselaer Polytechnic Institute - had petitioned to join 
the Institute if DOE had renewed funding for the program for another three years. This growth in faculty was clear evidence of the high level of interest and enthusiasm generated by the program in its first three years. The additional breadth that was added to the research portfolio enhanced the programmatic offerings for the fellows, as and enabled the Institute to address additional research areas. The addition of five faculty in the second three years was geared to establish and maintain a critical mass of participants at institutions other than Johns brought a specific new dimension to the program by providing expertise in high performance computing research resources, both human and hardware, as well as instructional facilities - as well as by involving faculty with experience using high performance computing as a research tool for computational biology.

During the initial three-year funding period, IMMBI grew to include 21 pre-doctoral fellows, 8 post-doctoral fellows. The high quality of the applicant pool of pre-doctoral fellows and the exceptionally high acceptance rate exceeded our expectations. Details of our recruiting activities are described in our first Annual Progress Report (April 2005). A distinguishing feature of the pre-doctoral fellows is the diversity of their educational backgrounds in science and engineering, as seen in the following list of the pre-doctoral fellows with their undergraduate affiliations and degrees:

Tural Aksel, Sabanci University
BS Bioengineering
Srinivas Aripirala, IIT Khangupar
BS Mathematics and Computing
(double major)
Violeta Beleva, Kent State University
BS Physics and Applied Math (double major)
MS Physics
Hao Chang, Tsinghua University
BS Applied Physics and MS Physics
Seung-Wook Chung, Seoul National Univ.
BS/MS Chemical Engineering
Eva Cunha, Universidade de Lisboa
BS Biochemistry and Bioinformatics
Ignacia Echeverria, U. Notre Dame
BS Physics

Tural Aksel, Sabanci University

Srinivas Aripirala, IIT Khangupar BS Mathematics and Computing

Violeta Beleva, Kent State University BS Physics and Applied Math (double major)

Hao Chang, Tsinghua University

Seung-Wook Chung, Seoul National Univ. BS/MS Chemical Engineering BS Physics

\author{
Sabri Erdemli, KOC University \\ BS Chemical Engineering \\ MS Computational Sciences \& Engineering \\ Kshitiz Gupta, IIT Bombay \\ BS Computer Science and Engineering \\ Yee Seir Kee, Luther College \\ BS Physics and Biology (double major) \\ Victor Khangulov, St. Mary's College of MD \\ BA Chemistry and Computer Science \\ (double major) \\ Anu Nagarajan, Stella Maris College. \\ BS Physics and MS Bioinformatics \\ Scott Patterson, Purdue University \\ BS Computer Science \& Mathematics \\ Juan Perilla, Universidad Nacional de Colombia \\ BA Physics
}

\author{
Chris Poirier, University of New Brunswick \\ BS Electrical Engineering \\ Hamsa Priya, Jawaharlal Nehru Tech Univ. \\ BS/MS Chemical Engineering \\ Asawari Samant, University of Bombay \\ BS Chemical Engineering \\ Grant Schauer, University of New Mexico \\ BS Biochemistry \\ Anne Marie Schilthuis, Penn State University \\ BS Chemical Engineering
An-Chi Wei, Georgetown University
MS Biochemistry \& Molecular Biology
Tzulan Yeh, Tsinghua University BS Engineering \& Systems Science MS Physics \\ BS Pharmacy
}

We also recruited a talented group of eight post-doctoral fellows for the specific goal to initiate new research collaborations between IMMBI faculty. The post-doctoral fellows are listed below with their Ph.D. research disciplines, academic affiliations, faculty co-mentors, and a brief statement of their research project.

- Olga Lubman, Ph.D. in Biochemistry and Molecular Biophysics from Washington University, worked in the Barrick and Levchenko labs. She studied Notch signaling pathways at multiple scales using a variety of computational and experimental approaches. 
- Erdinc Atilgan, Ph.D. in Physics from the University of Southern California, worked in the Sun and Schafer labs. He used molecular continuum models of actin-driven cell motility to describe actin network patterns observed during motility and explain their origins.

- Angela Liu, Ph.D. in Computational Chemistry from Carnegie Mellon University, worked in the Bader and Gray labs. She applied course-grained statistical models and all-atom molecular simulations to study DNA recognition by transcription factors

- Jay Jeong, Ph.D. in Mechanical and Aerospace Engineering from Seoul National University, worked in the Chirikjian and Rose labs. He applied coarse-grained normal mode analysis and molecular simulations to study contributions by dynamics to the stability and function of thermophilic proteins.

- Nick Panasik, Ph.D. in Biochemistry and Molecular Biology from Penn State University, worked in the Rose and Chirikjian labs. He used computational methods of directed evolution to study the specificity of folds of proteins.

- Dilip Asthagiri, Ph.D. in Chemical Engineering from the University of Delaware, worked in the Paulaitis and Pratt labs. He applied ab initio and classical molecular dynamics simulations and continuum models to study interactions between metal ions and peptides.

- Nick Fitzkee, Ph.D. in Biophysics from Johns Hopkins, worked in the Garcia-Moreno and Rose labs. He used continuum electrostatics and statistical thermodynamic models to study the character of electrostatic forces in unstructured and unfolded proteins.

- Jindal Shah, Ph.D. in Chemical Engineering from the University of Notre Dame, worked in the Paulaitis, Pratt, and Asthagiri labs. He applied statistical thermodynamic theory and molecular dynamics simulations to study the effects of hydration on peptide binding to proteins and peptide-mediated protein-protein interactions.

During the three years of DOE funding, these pre-doctoral and post-doctoral fellows have coauthored 27 research papers that have been published in scientific journals to date. In addition, they have contributed research results to 18 presentations or invited talks given at national and international meetings through the first three years of the program. Lists of these publications and presentations plus invited talks are given below. The substantial number of publications in the initial stages of this program and the quality of the scientific journals in which they appear reflect the high productivity of the IMMBI research enterprise, as well as the high quality of the research that is continues in the Institute without DOE support.

\section{Research Publications Co-Authored by IMMBI Fellows}

(IMMBI fellows are underlined.)

1. Jeong, J. I., Y. Jang, and M. K. Kim, 2005. "A connection rule for alpha-carbon coarsegrained elastic network models using chemical bond information," J. Mol. Graph. Model. 24:296.

2. Panasik, N., P. J. Fleming, and G. D. Rose, 2005. "Hydrogen-bonded turns in proteins: The case for a recount," Protein Sci. 14:2910.

3. Samant, A. and D. G. Vlachos, 2005. "Overcoming stiffness in stochastic simulation stemming from partial equilibrium: A multiscale Monte Carlo algorithm," J. Chem. Phys. 123:144114-1. 
4. Asthagiri, D., L. R. Pratt, and M. E. Paulaitis, 2006. "Role of fluctuations in a snug-fit mechanism of KcsA channel selectivity," J. Chem. Phys. 125:04701.

5. Atilgan, E., D. Wirtz, and S. X. Sun, 2006. "Mechanics and Dynamics of Actin-Driven Thin Membrane Protrusions," Biophys. J. 90:65.

6. Bose, R., H. Molina, A. S. Patterson, J. K. Bitok, B. Periaswamy, J. S. Bader, A. Pandey, and P. A. Cole, 2006. "Phosphoproteomic analysis of Her2/neu signaling and inhibition," Proc. Natl. Acad. Sci. 103:9773.

7. Fitzkee, N. C., P. J. Gong, P. J. Fleming, N. Panasik, T. O. Street, and G. D. Rose, 2006. "Are proteins made from a limited parts list?" Trends Biochem. Sci. 30:73.

8. Jeong, J. I., E. E. Lattman, and G. S. Chirikjian, 2006. "A method for finding candidate conformations for molecular replacement using relative rotation between domains of a known structure," Acta Crystallogr. D Biol. Crystallogr. 62:398.

9. Liu, L. A. and J. S. Bader, 2006. "Decoding transcriptional regulatory interactions," Physica D 224:174.

10. Paliwal, A., D. Asthagiri, L. R. Pratt, H. S. Ashbaugh and M. E. Paulaitis, 2006. "An analysis of molecular packing and chemical association in liquid water using quasichemical theory," J. Chem. Phys. 124:224502.

11. Atilgan, E., S. X. Sun, 2007. "Shape transitions in lipid membranes and protein mediated vesicle fusion and fission," J. Chem. Phys. 126:095102.

12. Lubman, O. Y., M. X. G. Ilagan, R. Kopan, and D. Barrick, 2007. "Quantitative Dissection of the Notch:CSL Interaction: Insights into the Notch-Mediated Transcriptional Switch" J. Mol. Biol. 365:577.

13. Chatterjee, A. and D.G. Vlachos, 2007. "An overview of spatial microscopic and accelerated kinetic Monte Carlo methods," J. Comput-Aided Mater. Des. 14:253.

14. Chatterjee, A. and D.G. Vlachos, 2007. "Continuum mesoscopic framework for multiple interacting species and processes on multiple site types and/or crystallographic planes," $J$. Chem. Phys. 127:034705.

15. Chatterjee, A. and D.G. Vlachos, 2007. "Systems tasks in nanotechnology via hierarchical multiscale modeling: Nanopattern formation in heteroepitaxy," Chem. Eng. Sci. 62:4852.

16. Asthagiri, D., Ashbaugh, H.S., Piryatinski, A., Paulaitis, M.E., and L. R. Pratt, 2007. "Nonvan-der-Waals treatment of the hydrophobic solubilities of $\mathrm{CF}_{4}$," J. Am. Chem. Soc. 129:10133.

17. Niehaus, A.-M.S., Vlachos, D.G., Edwards, J.S., Plechac, P., and R. Tribe, 2007. "Microscopic Simulation of Epidermal Growth Factor Receptor Diffusion on Corralled Membrane Surfaces," Biophys. J., 94:1551.

18. Poirier, C.C. and P.A. Iglesias, 2007. "An integrative approach to understanding mechanosensation," Briefings in Bioinformatics, 8:258.

19. Samant, A., B. A. Ogunnaike, and D. G. Vlachos, 2007. "A Hybrid Multiscale Monte Carlo Algorithm (HyMSMC) to Cope with Disparity in Time Scales and Species Populations in Well-Mixed Reaction Networks," BMC Bioinformatics, 8:175.

20. Shah, J. K., Asthagiri, D., Pratt, L.R., and M. E. Paulaitis, 2007. "Balancing local order and long-ranged interactions in the molecular theory of liquid water," J. Chem. Phys. 127:144508.

21. Collins, S. D., A. Chatterjee, and D.G. Vlachos, 2008. "Coarse-grained kinetic Monte Carlo models: Complex lattices, multicomponent systems, and homogenization at the stochastic level," J. Chem. Phys. 129:184101. 
22. Priya, M. H., J. K. Shah, D. Asthagiri, and M. E. Paulaitis, 2008. "Distinguishing thermodynamic and kinetic views of the preferential hydration of protein surfaces," Biophys. J. 95:2219.

23. Chung S. W., F. L. Miles, R. A. Sikes, C. R. Cooper, M. C. Farach-Carson, and B. A. Ogunnaike, 2009. "Quantitative Modeling and Analysis of the Transforming Growth Factor beta Signaling Pathway," Biophys. J. 96:1733.

24. Aksel, T. and D. Barrick, 2009. "Analysis of repeat-protein folding using nearest-neighbor statistical mechanical models," Methods in Enzymology: Biothermodynamics 455:95.

25. Castaneda, C. A., C. A. Fitch, A. Majumdar, V. Khangulov, J. L. Schlessman, and B. E. Garcia-Moreno, 2009. "Molecular determinants of the $\mathrm{pK}(\mathrm{a})$ values of Asp and Glu residues in staphylococcal nuclease," Proteins: Struc. Func. Bioinf. 77:570.

26. Beckstein, O., E. J. Denning, J. R. Perilla, and T. B. Woolf, 2009. "Zipping and Unzipping of Adenylate Kinase: Atomistic Insights into the Ensemble of Open <-> Closed Transitions," J. Mol. Biol. 394:160.

27. Zhou, L., S. Cortassa, A. C. Wei, M. A. Aon, R. L. Winslow, and B. O'Rourke B, 2009. "Modeling Cardiac Action Potential Shortening Driven by Oxidative Stress-Induced Mitochondrial Oscillations in Guinea Pig Cardiomyocytes," Biophys. J. 97:1843.

One of the primary goals of IMMBI has been to foster interdisciplinary collaborations among the faculty and their research groups by cultivating new research collaborations, and broadening the scope of long-standing collaboration. Faculty collaborations have involved graduate students or postdoctoral fellows who participated in the IMMBI program, but were supported from other sources, which allowed us to leverage DOE funding for the Institute. Dr. Damjanovic provides one example of how this worked. She was an NIH-funded postdoctoral fellow co-mentored by Professors Garcia-Moreno at Johns Hopkins and García at Rensselaer Polytechnic Institute who published three research papers as a result of this IMMBI research collaboration:

28. Damjanovic, A., B. Garcia-Moreno E., E. E. Lattman, and A. E. García, 2005. "Molecular dynamics study of hydration of the protein interior," Computer Phys. Communications. 169:126.

29. Damjanovic, A., B. García-Moreno E., E. E. Lattman, and A. E. García, 2005. "Molecular Dynamics Study of Water Penetration in Staphylococcal Nuclease," Proteins: Struc. Func. Bioinf. 60:433.

30. Damjanovic, A., J. Schlessman, F. A. Fitch, A. E. García, and B. Garcia-Moreno E., 2007. "Role of flexibility and polarity as determinants of the hydration of internal cavities and pockets in proteins," Biophys. J., 93:2791.

Another example was the post-doctoral fellowship for Asthagiri, which was funded jointly by IMMBI and Los Alamos National Laboratory with the goal to strengthen interactions between researchers at LANL and IMMBI. Following his postdoctoral appointment, Dr. Asthagiri was hired as an Assistant Professor at Johns Hopkins, which had the desired effect of consolidating several existing research collaborations among an even larger group of IMMBI faculty at the University Delaware (Lenhoff), Ohio State University (Paulaitis), LANL (Pratt), and Hopkins (Asthagiri). 
Presentations and Invited Talks on Research Conducted by IMMBI Fellows

(IMMBI fellows are underlined.)

1. Atilgan, E. and S. X. Sun, "Dynamics of Actin in Filopodia, and the Network Organization in Lamellipodia," Annual Meeting of the Biophysical Society, February 2005.

2. Jeong, J. I. and M. K. Kim, "A chemical bond based elastic network model in proteins," Annual Meeting of the Biophysical Society, February 2005.

3. Lubman, O. Y. and D. Barrick "In Vitro Dissection of Notch/RBPjk Interaction: Insights into Notch-Mediated Transcriptional "Switch," The $19^{\text {th }}$ Annual Gibbs Conference on Biothermodynamics, October 2005.

4. Samant, A., A. Chatterjee, and D. G. Vlachos, "Multiscale Stochastic Simulations of the Mitogen Activated Protein (MAP) Kinase Cascade," Annual Meeting of the American Institute of Chemical Engineers, November 2005.

5. Paulaitis, M. E., D. Asthagiri, A. Paliwal, D. Abras, and A. M. Lenhoff, "Role of Hydration in Weak Protein-Protein Interactions," Interfacial Phenomena at Different Length and Time Scales. International Chemical Congress of Pacific Basin Societies (Pacifichem), December 2005.

6. Asthagiri, D., L. R. Pratt, and M. E. Paulaitis, "Roles of fluctuations and a snug fit in KcsA channel selectivity," Annual Meeting of the Biophysical Society, February 2006.

7. Asthagiri, D., Pratt, L.R., and M. E. Paulaitis, "Role of fluctuations and a snug fit in KcsA channel selectivity," Annual Meeting of the American Institute of Chemical Engineers, November 2006.

8. Niehaus, A.-M.S., Vlachos, D.G. and J. S. Edwards, "Microscopic and Coarse Grained Stochastic Simulation of Epidermal Growth Factor Receptor Diffusion on Corralled Membrane Surfaces," Annual Meeting of the American Institute of Chemical Engineers, November 2006.

9. Samant, A., Vlachos, D.G., and B. A. Ogunnaike, "Hybrid, Multiscale Algorithm for Simulating Stochastic Systems," Annual Meeting of the American Institute of Chemical Engineers, November 2006.

10. Shah, J. K., Asthagiri, D., Pratt, L.R., and M. E. Paulaitis, "Computing Free Energies of Bound Water in Confined Environments and at Interfaces," Annual Meeting of the American Institute of Chemical Engineers, November 2006.

11. Shah, J. K., Asthagiri, D., and M. E. Paulaitis, "Computing Free Energies in PeptideMediated Protein-Protein Interactions in Modeling Immune Response," Annual Meeting of the American Institute of Chemical Engineers, November 2006.

12. Iglesias, P., Workshop on the Control of Uncertain Systems: Modelling, Approximation, and Design, Cambridge, UK, April 2006.

13. Iglesias, P., Feedback and Dynamics in Biology Colloqium. University of Washington, Seattle, May 2006.

14. Iglesias, P., Workshop on Predictive Models of Complex Systems. Washington University, Friday Harbor Labs, June 2006.

15. Iglesias, P., Institute of Nicolás Cabrera, 2006 Summer School on Biophysics of Biological Circuits: From Molecules to Networks, Miraflores de la Sierra (Madrid, Spain) September 2006.

16. Iglesias, P., Mathematics for Engineers Summer School: Mathematics for Robust and Nonlinear Control Systems, Leicester University, United Kingdom, September 2006.

17. Iglesias, P., Institute for Advanced Biosciences, Keio University, Japan. October 2006. 
18. Iglesias, P., Seventh International Conference on Systems Biology, Yokohama, Japan, October 2006.

19. Vlachos, D., "Multiscale model-based process and product engineering," Imperial College, London, UK, March 2006.

20. Vlachos, D., "The emerging field of multiscale simulation: Relation to cyber-infrastructure and educational needs," NSF Workshop on Cyber-based Combustion Science, Arlington, VA, April 2006.

21. Vlachos, D., "The emerging field of multiscale simulation in the biological sciences," Los Alamos National Laboratories, May 2006.

22. Vlachos, D., "Hierarchical multiscale model-based design of experiments, catalysts, and reactors for portable power generation," University of Pennsylvania, October 2006.

23. Vlachos, D., "The emerging field of multiscale simulation in the chemical, materials, and biological sciences," School of Chemical, Biological, and Materials Engineering, The University of Oklahoma, October 2006.

24. Vlachos, D., "The emerging field of multiscale simulation in the chemical, materials, and biological sciences," University of Wisconsin-Madison, Chemical \& Biological Engineering Department, October 2006.

25. Vlachos, D., "The emerging field of multiscale simulation in the chemical, materials, and biological sciences," Stevens, March 2007.

26. Vlachos, D., "The emerging field of multiscale simulation in the chemical, materials, and biological sciences," University of Florida, 2007.

27. Vlachos, D., "The emerging field of multiscale simulation: Application to scale-up of nanotechnological processes and control of cellular engineering," Department of Chemical and Biological Engineering, Tufts University, April 2007.

28. Iglesias, P., Gordon Research Conference on Gradient Sensing and Directed Cell Migration, Ventura, January 2007.

29. Fitzkee, N. and B. Garcia-Moreno E., "Modeling Electrostatic Properties of Unfolded Staphylococcal Nuclease," Annual Meeting of the Biophysical Society, March 2007.

30. Fitzkee, N., George Rose, and B. Garcia- Moreno E., "How random are random coils? Quantifying the amount of disorder in the unfolded state," Protein Society Meeting, July, 2007.

31. Fitzkee, N. and B. Garcia-Moreno E., "Electrostatic effects in unfolded proteins," American Chemical Society meeting on protein folding, Boston, August 2007.

32. Hamsa Priya, M., Asthagiri, D., and M. E. Paulaitis, "Bridging the Thermodynamic and the Kinetic Behavior of Waters of Protein Hydration," Annual Meeting of the Biophysical Society, March 2007.

33. Mayawala, K., Niehaus, A.-M.S., Vlachos, D.G., and J.S. Edwards, "Multiscale Stochastic Simulations for Systems Biology Modeling: Implications of Spatial Organization of EGF Receptor," MACKiE II meeting, February 2007.

34. Paulaitis, M. E., D. Asthagiri, and L. R. Pratt, "Role of Fluctuations in a Snug-Fit Mechanism of the KcsA K+ Channel Selectivity," Physical and Chemical Aspects of Molecular Biology. An International Workshop on Current Problems in Complex Fluids. Puebla, Mexico, January 2007.

35. Shah, J. K., Asthagiri, D., and M. E. Paulaitis, "Computing Free Energies of PeptideMediated Protein-Protein Interactions in Modeling the Immune System Response," Annual Meeting of the Biophysical Society, March 2007. 
Another notable accomplishment during the first three years of the program was the development of five new courses by IMMBI faculty designed to introduce pre-doctoral fellows to fundamental principles of biochemistry, cell and molecular biology, and to a broad range of computational methods for multiscale modeling of complex biological systems and processes:

- Biological Principles for Physical Scientists (Garcia-Moreno and Barrick) Introduction to modern biology at cellular and subcellular levels, focusing on molecular and cell biology and biochemistry. Course is for individuals with engineering and physical sciences background with little or no exposure to biology or chemistry. It is meant to give a basic biological framework for quantitative computational and physical studies of biological problems.

- Engineering Modeling and Analysis for Biological Systems (Paulaitis and Iglesias)

Introduction to multiscale models and simulations with an emphasis on surveying engineering methods for describing the behavior of biological systems and networks from cells to organs. The course objective is to familiarize students with the wide array of quantitative approaches applicable to biological computations within the context of multiscale modeling and analysis.

- Molecular Modeling of Biological Interactions (Paulaitis and Gray)

A survey course on quantitative analytical methods and computational approaches for modeling molecular interactions in biological systems. Topics include: $a b$ initio and semi-empirical quantum mechanical methods, molecular simulations, molecular models of protein-protein interactions, non-equilibrium statistical mechanics models of dynamics, computational mechanics of biological macromolecules, bioinformatics approaches to structure prediction, network analysis for protein-protein and protein-DNA interactions.

- Short Course on Multiscale Modeling and Simulations (Vlachos and Edwards)

A modular course on multiscale modeling and simulation related, but not limited, to biological systems. The modules are: Markov processes, averaging, coarse graining; hybrid multiscale simulation; multiscale aspects of biological systems; multiscale stochastic modeling; membrane biology; signal transduction pathways; rule-based modeling of biochemical systems. Lecture notes and videotapes are available at: $\underline{\mathrm{http}: / / \mathrm{www} . c h e . u d e l . e d u / c c s t}$

- Biological Control Systems (Ogunnaike)

This course explores the principles and methods by which the mammalian organism maintains stable, efficient and "near-optimal" performance, and homeostasis in the face of external and internal perturbations. The distinct biological systems responsible for this overall task range from physiological, to cellular, and sub-cellular. The course presents a control engineering perspective of the function, organization, and coordination of these multi-scale biological control systems.

The modeling courses were filmed and made web-accessible along with the course material so that fellows at all participating IMMBI institutions could view the lectures and study the course material. There were also many elective courses offered by the IMMBI faculty that were taken by postdoctoral and pre-doctoral fellows depending on their specific educational backgrounds needs, and interests:

- Computational Mechanics of Biological Macromolecules. (Chirikjian)

We examine numerical methods for modeling shape fluctuations in biomolecules using coursegrained elastic network models. No prior knowledge of biochemistry or molecular biology is 
required. Knowledge of linear algebra and differential equations is required. A basic understanding of Newtonian and Lagrangian dynamics will be helpful, but will be reviewed.

\section{- Introduction to Biomolecular and Nanoscale Simulation. (Gray)}

This course aims to teach fundamental concepts and techniques underyling the simulation of biomolecular and nonscale systems on the time and length scales that correspond to the assembly of components intro supramolecular structures. We will examine the forces relevant at the nanoscale, motions experienced by biomacromolecules or other nanoscale components, and methodologies for capturing behavior efficiently.

- Molecular Simulations and Multiscale Modeling. (Gray and Drazer)

This course gives an introduction to modern numerical methods for calculating thermodynamics, transport and structural properties of complex systems in chemical and biomolecular engineering. The first part of the course will focus on molecular level simulations, their applications and limitations. The second part will emphasize the need for multiscale models to address the multiplicity of length and time scales in complex systems. Mesoscopic models will be investigated as a first step into multiscale models. Current methods to bridge the gap between atomistic simulations and macroscopic methods will be presented in case studies.

- Computer Modeling of Biological Macromolecules Lecture. (Amzel, Garcia-Moreno, Rose, Shortle and Woolf)

Lectures will offer an introduction to the mathematical aspects of computer representation and manipulation of macromolecules followed by discussions of important topics in the computational chemistry of macromolecules including: forces and potential fields, molecular mechanics, Monte Carlo methods and others. A laboratory course can be taken simultaneously as a complement.

- Computer Modeling of Biological Macromolecules Lab. (Bianchet and Amzel)

The laboratory will introduce users to the commercial package Quanta and to the public domain programs for modeling, energy minimization, docking and other techniques. The lecture course can be taken simultaneously.

- Biostatistics for Laboratory Scientists. (Ruczinski)

Introduces the basic concepts and methods of statistics with applications in the experimental biological sciences.

- Proteins and Nucleic Acids. (Rose)

This course emphasizes structural thermodynamics of proteins and nucleic acids. An accompanying laboratory component involves computer modeling with addition lectures on molecular modeling and genetic algorithms. Proteins as nature's molecular robots, and DNA/RNA, the genetic material. From genetic code to protein structure. Experimental approaches to understanding macromolecules, models, model-working, and computer simulations. Visualizing three-dimensional structures.

Proteins and Nucleic Acids. (Rose)

Proteins as nature's molecular robots, and DNA/RNA, the genetic material. From genetic code to protein structure. Experimental approaches to understanding macromolecules, models, modelworking, and computer simulations. Visualizing three-dimensional structures. 
- Physical Chemistry of Biological Macromolecules. (Garcia-Moreno)

This course emphasizes principles of thermodynamics, statistical thermodynamics and kinetics in the study o the physical basis of macromolecular structure. A substantial section is devoted to demonstrating how complex biological behavior such as the life cycle of bacteriophage lambda, can be understood baed on rigorous knowledge of the energetics and kinetics of interaction among the key participating macromolecules that regulate gene experession.

- Statistical Mechanics of Biological Systems. (Sun)

We discuss the dynamics of many-body systems and draw general conclusions that motivat the basic principles of statistical mechanics. The connection between statistical mechanics with thermodynamics is made, and applications to proteins and membranes are presented. Computer simulations of these systems are discussed extensively. Finally, we examine some models of non-equilibrium statistical mechanics and apply them to ion channels and molecular motors.

- Structure and Chemistry of Lipid Bilayers. (Woolf)

This course focuses on the structure, physical properties, and chemistry of lipids and lipid membranes. Methods for studying lipid membranes will be discussed in detail, including experimental approaches, theoretical (analytical) calculations, and computational methods. Complex behavior of lipid membranes, including pore formation and membrane fusion, and the interaction of ions and water with membranes will also be examined.

- Fundamentals of Membrane Physiology. (Woolf)

This course focuses on the foundations of modern membrane physiology. General concepts for understanding potentials across membranes, including the Nernst equation and the HodkinHuxley formalism, will be presented, followed by a detailed discussion of ion channels and membrane transporters. Pathological conditions associated with ion channel or transporter malfunction and advances in network analysis of membrane signaling will also be covered.

- Feedback Control of Biological Signaling Pathways. (Iglesias)

This course examines the use of feedback control in engineering systems and searches for counterparts in biological signaling networks. Some knowledge of mathematical modeling techniques in biology is required, so a part of the course will be devoted to introducing dynamical systems theory and control, and modeling biochemical networks.

- Systems Biology of Cell Regulation. (Levchenko)

Recent advances in Systems Biology research of intracellular processes are explored in depth through extensive review and analysis of the corresponding computational models and experimental techniques. Examples include modeling and experimental studies of metabolic, genetic, signal transduction and cell cycle regulation networks. The classes alternate between consideration of network-driven and network element-driven approaches.

- Feedback control in biological signaling pathways. (Iglesias)

Biological signaling pathways are composed of large "networks" of interconnected molecular components, many of which involve considerable feedback and are highly nonlinear. The subsystems comprising the network are subject to control by many independent events, and thus defy attempts to describe clear cut cause-and-effect relationships. Because of these features, the complete understanding of how these systems work presents a problem of daunting complexity to researchers. Feedback control theory is commonly used in engineering practice as a means of regulating man-made systems. In this course we study biological signaling pathways using the tools of control systems. 
- Protein Bioinformatics. (Ruczinski)

This course provides students with an overview of protein bioinformatics including computational and experimental approaches.

- Chemical Engineering Principles I. (Vlachos)

This three-credit course covers basics of linear algebra and differential equations. One module of the course focuses on time scale analysis of ordinary differential equations and algebraic equations. Topics among others include dimensional analysis, sensitivity analysis, flux analysis, principal component analysis, regular and singular perturbation methods, computational singular perturbation, low dimensional manifold theory, matched asymptotics, computer-aided model reduction. This material forms the backbone of multiscale analysis of deterministic models.

- Chemical Engineering Principles II. (Vlachos)

This four-credit course is modular with typical modules including partial differential equations and tensor analysis, stochastic modeling, design of experiments, probability and statistics. In one of the modules, Vlachos focuses on stochastic Monte Carlo simulation along with its temporal acceleration, such as t-leap, stochastic low dimensional manifolds and computational singular perturbation, and Langevin dynamics. In both courses, several biological examples are presented.

Additional programmatic accomplishments during the first three years include:

(1) IMMBI annual retreats held in November 2005 and 2006.

(2) Inaugural IMMBI Lectures presented by Professor Douglas Lauffenburger, MIT Biological Engineering Division and Computational \& Systems Biology Initiative in October 2005, and Professor Ken Dill, Department of Pharmaceutical Chemistry, University of California, San Francisco in October 2006.

(3) Co-sponsored departmental seminars by Dr. Saroja Ramanujan (Entelos Inc.), Professor Roman Osman (Mount Sinai), Professor Ivet Bahar (University of Pittsburgh), Professor George Oster (University of California, Berkeley), Professor Qiang Cui (University of Wisconsin, Madison), Professor Bruce Tidor (MIT), Professor Arieh Warshel (University of Southern California).

Structure of the Training Program

The training program requirements were established during the three-year start-up of the Institute as outlined below, which included some minor modifications to take into account special cases for individuals at institutions other than Johns Hopkins because of the smaller numbers of faculty and fellows involved.

IMMBI fellows were required to demonstrate proficiency in biochemistry, molecular and cell biology, and in engineering analysis, numerical methods, and simulations. Lecture courses were offered to provide the necessary instruction in these areas, and student enrolment for these courses were determined on a case-by-case basis depending on their educational backgrounds. These courses were co-listed at the participating institutions, and distributed via the distance learning capabilities available at these institutions. Course material was also web-accessible to all IMMBI fellows and faculty.

Pre-doctoral fellows were required to have two faculty co-mentors to reflect complementary multiscale modeling/simulation and analysis components to the proposed doctoral research. In an external review of the IMMBI program by DOE staff at the end of second year, the review 
panel recommended that the selection of research co-mentors and projects also allow for an experimental component to be included in some cases. Therefore, pre-doctoral fellows were permitted to design a collaboration of faculty co-mentors in which one would contribute a multiscale modeling/simulation and analysis component and the other would contribute an experimental component to the research.

It was our experience that the requirement of dual mentors is the most effective way to promote faculty interactions and long-term collaborations. In addition, the research progress of every predoctoral fellow was reviewed annually by an IMMBI research review committee. This review committee consisted of three standing faculty members, faculty co-mentors, and any additional faculty members deemed by the review committee to be appropriate for the fellow and his or her research project. This annual review also contributed to promoting faculty collaborations.

Curricular requirements in the training program were kept to a minimum to allow pre-doctoral fellows to pursue a curriculum uniquely structured to individual needs and interests. This philosophy worked well for the first two classes of IMMBI pre-doctoral fellows, especially in light of their diverse educational backgrounds.

Eight post-doctoral fellows were recruited into IMMBI during the initial three-year funding cycle with the primary goal to foster research collaborations among the IMMBI faculty. In many cases, the fellowships did successfully initiate new collaborations among the faculty. We concluded, however, that the initial two-year commitment to pre-doctoral fellows with the requirement of faculty co-mentors provides a better vehicle for fostering research collaborations compared to the one-year postdoctoral fellowships.

Program integration was an important consideration in the initial three-year funding cycle, since IMMBI fellows were distributed across different academic departments on several campuses at multiple institutions. Numerous steps were taken to avoid the isolation, fragmentation, or simple disorganization in this environment. For example, academic advising for all pre-doctoral fellows was provided by a select group of IMMBI faculty during their first two years in the program. IMMBI funding was also provided funding to allow pre-doctoral fellows to spend a semester in residence in a faculty research laboratory at another institution. A full-time academic coordinator for the Institute distributed information about activities in the different academic departments and institutions - e.g., special seminars co-sponsored by IMMBI, or departmental seminars that might have been of special interest to IMMBI fellows and faculty - and coordinated various IMMBI activities, such the annual retreat and the Inaugural Lectures. Oral presentations of work accomplished in the laboratory rotations also brought together IMMBI fellows and faculty.

During its three-year start-up, the Institute was formally directed by Professor Paulaitis, and codirected by Professors Garcia-Moreno at Hopkins and Lenhoff at Delaware. In practice, all three served as co-directors when Professor Paulaitis moved to Ohio State University, although he did maintain joint appointments in Chemical and Biomolecular Engineering and Biophysics at Hopkins, and commuted biweekly between Ohio State and Hopkins. Professor Iglesias was added as an Institute co-director in the second year of the program. The IMMBI Steering Committee consisted of Professors Chirikjian, Amzel, Barrick, Woolf and the co-directors Professors Garcia-Moreno, Paulaitis, Lenhoff, and Iglesias - was also established and functioned to set the policies and the direction of the Institute. 
Because the Institute spanned multiple institutions, a highly centralized decision making process was neither practical nor desirable. The main responsibilities of the co-directors, Professors Paulaitis but primarily Garcia-Moreno and Iglesias at Hopkins and Professor Lenhoff at Delaware were to carry out the policies of the Institute on a day-to-day basis. In addition, IMMBI faculty members located on other campuses served as additional academic advisors to the fellows in this program, and as such, have monitored academic progress and addressed minor issues in an informal and more personal way. We found this management structure worked well.

The first formal, external review of the IMMBI program was conducted on May 24, 2006. The external review panel consisted of Drs. Anil Deane, Christine Chalk, and John Houghton from DOE, and Professors Julie Mitchell and Michael Colvin, Directors of the two other DOE-funded Institutes at the University of Wisconsin and the University of California-Merced, respectively. Based on the constructive assessment we received about the program at that time, plans were put in place to expand the review panel was to include faculty from other universities and scientific leaders in computational biology at industrial laboratories. 